Research Article; Received: March 26, 2021; Accepted: June 1, 2021

\title{
LACUNARY INVARIANT STATISTICAL EQUIVALENCE FOR DOUBLE SET SEQUENCES
}

\author{
Uğur ULUSU ${ }^{1}$, Erdinç DÜNDAR ${ }^{2}$, and Nimet PANCAROĞLU AKIN ${ }^{2}$ \\ ${ }^{1}$ Sivas Cumhuriyet University, 58140 Sivas, TURKEY \\ ${ }^{2}$ Afyon Kocatepe University, 03200 Afyonkarahisar, TURKEY
}

\begin{abstract}
In this paper, we introduce the notions of asymptotical strong $\sigma_{2}$-equivalence, asymptotical $\sigma_{2}$-statistical equivalence, asymptotical lacunary strong $\sigma_{2}$-equivalence and asymptotical lacunary $\sigma_{2}$-statistical equivalence in the Wijsman sense for double set sequences. Also, we investigate some relations between these new asymptotical equivalence notions.
\end{abstract}

\section{INTRODUCTION}

Long after the notion of convergence for double sequences was introduced by Pringsheim 1], this notion was extended to the notion of statistical convergence by Móricz [2] and Mursaleen and Edely [3] in the same year, to the notion of lacunary statistical convergence by Patterson and Savaş [4] and to the notion of double $\sigma$ convergent lacunary statistical sequence by Savaş and Patterson [5]. Moreover, for double sequences, the notion of asymptotical equivalence was introduced by Patterson [6].

Over the years, on the various convergence notions for set sequences have been studied by many authors (see, $[7[\sqrt[9]{9}$ ). One of them, discussed in this paper, is the notion of convergence in the Wijsman sense $[10$. Using the notions of statistical convergence, double lacunary sequence and invariant mean, this notion was extended to the notions of convergence for double set sequences by some authors $11-13$. Furthermore, for double set sequences, the notions of asymptotical equivalence in the Wijsman sense were introduced by Nuray et al. 14 and then these notions were studied by some authors $15-17$. In this paper, using the notion of invariant

2020 Mathematics Subject Classification. Primary 34C41; Secondary 40A05, 40A35.

Keywords. Asymptotical equivalence, double lacunary sequence, invariant mean, statistical convergence, convergence in the Wijsman sense.

■ugurulusu@cumhuriyet.edu.tr-Corresponding author; edundar@aku.edu.tr; npancaroglu@aku.edu.tr

(D) 0000-0001-7658-6114; 0000-0002-0545-7486; 0000-0003-2886-3679.

Communications 
mean, we study on new asymptotical equivalence notions in the Wijsman sense for double set sequences. More information on the notions of asymptotical equivalence for set sequences can be found in 18,19.

\section{Basic Definitions and Notations}

In this section, let us remind the basic notions necessary for a better understanding of our paper.

Definition 1. [1] A double sequence $\left(x_{j k}\right)$ is called convergent to $L$ in Pringsheim's sense if for every $\varepsilon>0$, there exists $N_{\varepsilon} \in \mathbb{N}$ such that $\left|x_{j k}-L\right|<\varepsilon$, whenever $j, k>N_{\varepsilon}$. It is denoted by $P-\lim _{j, k \rightarrow \infty} x_{k j}=L$ or $\lim _{j, k \rightarrow \infty} x_{j k}=L$.

Definition 2. [3] A double sequence $\left(x_{j k}\right)$ is called statistically convergent to $L$ if for every $\varepsilon>0$,

$$
P-\lim _{m, n \rightarrow \infty} \frac{1}{m n}\left|\left\{(j, k): j \leq m, k \leq n,\left|x_{j k}-L\right| \geq \varepsilon\right\}\right|=0 .
$$

For a metric space $(Y, d), \mu(y, B)$ denote the distance from $y$ to $B$ where

$$
\mu(y, B)=\inf _{b \in B} d(y, b)
$$

for any $y \in Y$ and any nonempty $B \subseteq Y$.

Throughout this study, $(Y, d)$ will be considered as a metric space and $B, B_{j k}, D_{j k}$ will be considered as any nonempty closed subsets of $Y$.

Definition 3. [13] A double set sequence $\left\{B_{j k}\right\}$ is called convergent to the set $B$ in the Wijsman sense if for each $y \in Y$,

$$
P-\lim _{j, k \rightarrow \infty} \mu\left(y, B_{j k}\right)=\mu(y, B) .
$$

Let $\sigma$ be a mapping such that $\sigma: \mathbb{N} \rightarrow \mathbb{N}$ (the set of positive integers). A continuous linear functional $\psi$ on $\ell_{\infty}$, the space of real bounded sequences, is called an invariant mean (or a $\sigma$-mean) if it satisfies the following conditions:

(1) $\psi\left(x_{s}\right) \geq 0$, when the sequence $\left(x_{s}\right)$ has $x_{s} \geq 0$ for all $s$,

(2) $\psi(e)=1$, where $e=(1,1,1, \ldots)$ and

(3) $\psi\left(x_{\sigma(s)}\right)=\psi\left(x_{s}\right)$ for all $\left(x_{s}\right) \in \ell_{\infty}$.

The mapping $\sigma$ is assumed to be one-to-one and such that $\sigma^{j}(s) \neq s$ for all $j, s \in \mathbb{N}$, where $\sigma^{j}(s)$ denotes the $j$ th iterate of the mapping $\sigma$ at $s$. Thus $\psi$ extends the limit functional on $c$, the space of convergent sequences, in the sense that $\psi\left(x_{s}\right)=\lim x_{s}$ for all $\left(x_{s}\right) \in c$.

Definition 4. [12] A double set sequence $\left\{B_{j k}\right\}$ is called invariant convergent to the set $B$ in the Wijsman sense if for each $y \in Y$,

$$
P-\lim _{n, m \rightarrow \infty} \frac{1}{n m} \sum_{j, k=1,1}^{n, m} \mu\left(y, B_{\sigma^{j}(s) \sigma^{k}(t)}\right)=\mu(y, B), \quad \text { uniformly in } s, t \text {. }
$$


Definition 5. [12] A double set sequence $\left\{B_{j k}\right\}$ is called strong invariant convergent to the set $B$ in the Wijsman sense if for each $y \in Y$,

$$
P-\lim _{n, m \rightarrow \infty} \frac{1}{n m} \sum_{j, k=1,1}^{n, m}\left|\mu\left(y, B_{\sigma^{j}(s) \sigma^{k}(t)}\right)-\mu(y, B)\right|=0, \quad \text { uniformly in s, } t .
$$

Definition 6. [12] $A$ double set sequence $\left\{B_{j k}\right\}$ is called invariant statistically convergent to the set $B$ in the Wijsman sense if for every $\varepsilon>0$ and each $y \in Y$,

$$
P-\lim _{n, m \rightarrow \infty} \frac{1}{n m}\left|\left\{(j, k): j \leq n, k \leq m,\left|\mu\left(y, B_{\sigma^{j}(s) \sigma^{k}(t)}\right)-\mu(y, B)\right| \geq \varepsilon\right\}\right|=0,
$$

uniformly in $s, t$.

A double sequence $\theta_{2}=\left\{\left(j_{r}, k_{u}\right)\right\}$ is called a double lacunary sequence if there exist increasing sequences $\left(j_{r}\right)$ and $\left(k_{u}\right)$ of the integers such that

$$
j_{0}=0, h_{r}=j_{r}-j_{r-1} \rightarrow \infty \text { and } k_{0}=0, \bar{h}_{u}=k_{u}-k_{u-1} \rightarrow \infty \text { as } r, u \rightarrow \infty .
$$

In general, the following notations is used for any double lacunary sequence:

$$
\begin{gathered}
h_{r u}=h_{r} \bar{h}_{u}, I_{r u}=\left\{(j, k): j_{r-1}<j \leq j_{r} \text { and } k_{u-1}<k \leq k_{u}\right\}, \\
q_{r}=\frac{j_{r}}{j_{r-1}} \text { and } q_{u}=\frac{k_{u}}{k_{u-1}} .
\end{gathered}
$$

Throughout this study, $\theta_{2}=\left\{\left(j_{r}, k_{u}\right)\right\}$ will be considered as a double lacunary sequence.

Definition 7. [12] A double set sequence $\left\{B_{j k}\right\}$ is called lacunary invariant convergent to the set $B$ in the Wijsman sense if for each $y \in Y$,

$$
P-\lim _{r, u \rightarrow \infty} \frac{1}{h_{r u}} \sum_{(j, k) \in I_{r u}} \mu\left(y, B_{\sigma^{j}(s) \sigma^{k}(t)}\right)=\mu(y, B), \quad \text { uniformly in } s, t .
$$

Definition 8. [12] A double set sequence $\left\{B_{j k}\right\}$ is called lacunary strong invariant convergent to the set $B$ in the Wijsman sense if for each $y \in Y$,

$$
P-\lim _{r, u \rightarrow \infty} \frac{1}{h_{r u}} \sum_{(j, k) \in I_{r u}}\left|\mu\left(y, B_{\sigma^{j}(s) \sigma^{k}(t)}\right)-\mu(y, B)\right|=0, \quad \text { uniformly in } s, t .
$$

Definition 9. [12] A double set sequence $\left\{B_{j k}\right\}$ is called lacunary invariant statistically convergent to the set $B$ in Wijsman sense if for every $\varepsilon>0$ and each $y \in Y$,

$$
P-\lim _{r, u \rightarrow \infty} \frac{1}{h_{r u}}\left|\left\{(j, k) \in I_{r u}:\left|\mu\left(y, B_{\sigma^{j}(s) \sigma^{k}(t)}\right)-\mu(y, B)\right| \geq \varepsilon\right\}\right|=0,
$$

uniformly in $s, t$. 
The term $\mu_{y}\left(\frac{B_{j k}}{D_{j k}}\right)$ is defined as follows:

$$
\mu_{y}\left(\frac{B_{j k}}{D_{j k}}\right)=\left\{\begin{array}{cc}
\frac{\mu\left(y, B_{j k}\right)}{\mu\left(y, D_{j k}\right)} & , \quad y \notin B_{j k} \cup D_{j k} \\
\lambda \quad, \quad y \in B_{j k} \cup D_{j k} .
\end{array}\right.
$$

Definition 10. 14, Two double set sequences $\left\{B_{j k}\right\}$ and $\left\{D_{j k}\right\}$ are called asymptotically equivalent of multiplicity $\lambda$ in the Wijsman sense if for each $y \in Y$,

$$
P-\lim _{j, k \rightarrow \infty} \mu_{y}\left(\frac{B_{j k}}{D_{j k}}\right)=\lambda .
$$

It is denoted by $B_{j k} \stackrel{W_{2}^{\lambda}}{\sim} D_{j k}$ and simply called asymptotically equivalent in the Wijsman sense if $\lambda=1$.

As an example to asymptotically equivalent double set sequences, the following sequences can be considered:

$$
B_{j k}=\left\{(a, b) \in \mathbb{R}^{2}: a^{2}+b^{2}-2 j k b=0\right\}
$$

and

$$
D_{j k}=\left\{(a, b) \in \mathbb{R}^{2}: a^{2}+b^{2}+2 j k b=0\right\} .
$$

Since

$$
P-\lim _{j, k \rightarrow \infty} \mu_{y}\left(\frac{B_{j k}}{D_{j k}}\right)=1
$$

for every $y \in \mathbb{R}^{2}$, the double set sequences $\left\{B_{j k}\right\}$ and $\left\{D_{j k}\right\}$ are asymptotically equivalent in the Wijsman sense, i.e., $B_{j k} \stackrel{W_{2}}{\sim} D_{j k}$.

\section{MAin RESUlts}

In this section, for double set sequences, we introduce the notions of asymptotical $\sigma_{2}$-equivalence, asymptotical strong $\sigma_{2}$-equivalence, asymptotical $\sigma_{2}$-statistical equivalence, asymptotical lacunary $\sigma_{2}$-equivalence, asymptotical strong lacunary $\sigma_{2}$-equivalence and asymptotical lacunary $\sigma_{2}$-statistical equivalence in the Wijsman sense. Also, we investigate some relations between some of these new asymptotical equivalence notions.

Definition 11. Two double set sequences $\left\{B_{j k}\right\}$ and $\left\{D_{j k}\right\}$ are said to be asymptotically $\sigma_{2}$-equivalent of multiplicity $\lambda$ in the Wijsman sense if for each $y \in Y$,

$$
P-\lim _{n, m \rightarrow \infty} \frac{1}{n m} \sum_{j, k=1,1}^{n, m} \mu_{y}\left(\frac{B_{\sigma^{j}}(s) \sigma^{k}(t)}{D_{\sigma^{j}}(s) \sigma^{k}(t)}\right)=\lambda, \quad \text { uniformly in } s, t .
$$

This type of equivalence is denoted by $B_{j k} \stackrel{W_{\sigma_{2}}^{\lambda}}{\sim} D_{j k}$ and simply called asymptotically $\sigma_{2}$-equivalent in the Wijsman sense if $\lambda=1$. 
Definition 12. Two double set sequences $\left\{B_{j k}\right\}$ and $\left\{D_{j k}\right\}$ are said to be asymptotically strong $\sigma_{2}$-equivalent of multiplicity $\lambda$ in the Wijsman sense if for each $y \in Y$,

$$
P-\lim _{n, m \rightarrow \infty} \frac{1}{n m} \sum_{j, k=1,1}^{n, m}\left|\mu_{y}\left(\frac{B_{\sigma^{j}(s) \sigma^{k}(t)}}{D_{\sigma^{j}(s) \sigma^{k}(t)}}\right)-\lambda\right|=0, \quad \text { uniformly in } s, t .
$$

This type of equivalence is denoted by $B_{j k} \stackrel{\left[W_{\sigma_{2}}^{\lambda}\right]}{\sim} D_{j k}$ and simply called asymptotically strong $\sigma_{2}$-equivalent in the Wijsman sense if $\lambda=1$.

The set of all asymptotically strong $\sigma_{2}$-equivalent double set sequences of multiplicity $\lambda$ in the Wijsman sense is denoted by $\left\{\left[W_{\sigma_{2}}^{\lambda}\right]\right\}$.

Definition 13. Two double set sequences $\left\{B_{j k}\right\}$ and $\left\{D_{j k}\right\}$ are said to be asymptotically $\sigma_{2}$-statistical equivalent of multiplicity $\lambda$ in the Wijsman sense if for every $\varepsilon>0$ and each $y \in Y$,

$$
P-\lim _{n, m \rightarrow \infty} \frac{1}{n m}\left|\left\{(j, k): j \leq n, k \leq m,\left|\mu_{y}\left(\frac{B_{\sigma^{j}(s) \sigma^{k}(t)}}{D_{\sigma^{j}(s) \sigma^{k}(t)}}\right)-\lambda\right| \geq \varepsilon\right\}\right|=0,
$$

uniformly in $s, t$. This type of equivalence is denoted by $B_{j k} \stackrel{W S_{\sigma_{2}}^{\lambda}}{\sim} D_{j k}$ and simply called asymptotically $\sigma_{2}$-statistical equivalent in the Wijsman sense if $\lambda=1$.

The set of all asymptotically $\sigma_{2}$-statistical equivalent double set sequences of multiplicity $\lambda$ in the Wijsman sense is denoted by $\left\{W S_{\sigma_{2}}^{\lambda}\right\}$.

Definition 14. Two double set sequences $\left\{B_{j k}\right\}$ and $\left\{D_{j k}\right\}$ are said to be asymptotically lacunary $\sigma_{2}$-equivalent of multiplicity $\lambda$ in the Wijsman sense if for each $y \in Y$,

$$
P-\lim _{r, u \rightarrow \infty} \frac{1}{h_{r u}} \sum_{(j, k) \in I_{r u}} \mu_{y}\left(\frac{B_{\sigma^{j}}(s) \sigma^{k}(t)}{D_{\sigma^{j}}(s) \sigma^{k}(t)}\right)=\lambda, \quad \text { uniformly in } s, t .
$$

This type of equivalence is denoted by $B_{j k} \stackrel{W_{\theta \sigma_{2}}^{\lambda}}{\sim} D_{j k}$ and simply called asymptotically lacunary $\sigma_{2}$-equivalent in the Wijsman sense if $\lambda=1$.

Definition 15. Two double set sequences $\left\{B_{j k}\right\}$ and $\left\{D_{j k}\right\}$ are said to be asymptotically lacunary strong $\sigma_{2}$-equivalent of multiplicity $\lambda$ in the Wijsman sense if for each $y \in Y$,

$$
P-\lim _{r, u \rightarrow \infty} \frac{1}{h_{r u}} \sum_{(j, k) \in I_{r u}}\left|\mu_{y}\left(\frac{B_{\sigma^{j}(s) \sigma^{k}(t)}}{D_{\sigma^{j}(s) \sigma^{k}(t)}}\right)-\lambda\right|=0, \quad \text { uniformly in } s, t .
$$

This type of equivalence is denoted by $B_{j k} \stackrel{\left[W_{\theta \sigma_{2}}^{\lambda}\right]}{\sim} D_{j k}$ and simply called asymptotically lacunary strong $\sigma_{2}$-equivalent in the Wijsman sense if $\lambda=1$. 
Example 1. Let $Y=\mathbb{R}^{2}$ and double set sequences $\left\{B_{j k}\right\}$ and $\left\{D_{j k}\right\}$ be defined as following:

$B_{j k}:=\left\{\begin{array}{cl}\left\{(a, b) \in \mathbb{R}^{2}: a^{2}+(b+1)^{2}=\frac{1}{j k}\right\} & ; \quad \text { if }(j, k) \in I_{r u}, j \text { and } k \text { are } \\ \text { square integers, } & \text { itherwise. }\end{array}\right.$

and

$D_{j k}:=\left\{\begin{array}{cl}\left\{(a, b) \in \mathbb{R}^{2}: a^{2}+(b-1)^{2}=\frac{1}{j k}\right\} & ; \text { if }(j, k) \in I_{r u}, j \text { and } k \text { are } \\ & \text { square integers, } \\ \{(2,0)\} & ; \text { otherwise. }\end{array}\right.$

In this case, the double set sequences $\left\{B_{j k}\right\}$ and $\left\{D_{j k}\right\}$ are asymptotically lacunary strong $\sigma_{2}$-equivalent in the Wijsman sense.

The set of all asymptotically lacunary strong $\sigma_{2}$-equivalent double set sequences of multiplicity $\lambda$ in the Wijsman sense is denoted by $\left\{\left[W_{\theta \sigma_{2}}^{\lambda}\right]\right\}$.

Definition 16. Two double set sequences $\left\{B_{j k}\right\}$ and $\left\{D_{j k}\right\}$ are said to be asymptotically lacunary $\sigma_{2}$-statistical equivalent of multiplicity $\lambda$ in the Wijsman sense if for every $\varepsilon>0$ and each $y \in Y$,

$$
P-\lim _{r, u \rightarrow \infty} \frac{1}{h_{r u}}\left|\left\{(j, k) \in I_{r u}:\left|\mu_{y}\left(\frac{B_{\sigma^{j}(s) \sigma^{k}(t)}}{D_{\sigma^{j}(s) \sigma^{k}(t)}}\right)-\lambda\right| \geq \varepsilon\right\}\right|=0,
$$

uniformly in $s, t$. This type of equivalence is denoted by $B_{j k} \stackrel{W S_{\theta \sigma_{2}}^{\lambda}}{\sim} D_{j k}$ and simply called asymptotically lacunary $\sigma_{2}$-statistical equivalent in the Wijsman sense if $\lambda=$ 1.

Example 2. Let $Y=\mathbb{R}^{2}$ and double set sequences $\left\{B_{j k}\right\}$ and $\left\{D_{j k}\right\}$ be defined as following:

$B_{j k}:=\left\{\begin{array}{cl}\left\{(a, b) \in \mathbb{R}^{2}:(a-j)^{2}+(b+k)^{2}=4\right\} & ; \quad \text { if }(j, k) \in I_{r u}, j \text { and } k \text { are } \\ \text { square integers, } \\ \{(-2,1)\} & ; \text { otherwise. }\end{array}\right.$

and

$D_{j k}:=\left\{\begin{array}{cl}\left\{(a, b) \in \mathbb{R}^{2}:(a+j)^{2}+(b-k)^{2}=4\right\} & ; \quad \text { if }(j, k) \in I_{r u}, j \text { and } k \text { are } \\ \text { square integers, } \\ \{(-2,1)\} & ; \text { otherwise. }\end{array}\right.$

In this case, the double set sequences $\left\{B_{j k}\right\}$ and $\left\{D_{j k}\right\}$ are asymptotically lacunary $\sigma_{2}$-statistical equivalent in the Wijsman sense.

The set of all asymptotically lacunary $\sigma_{2}$-statistical equivalent double set sequences of multiplicity $\lambda$ in the Wijsman sense is denoted by $\left\{W S_{\theta \sigma_{2}}^{\lambda}\right\}$. 


\section{Theorem 1.}

(i) If $B_{j k} \stackrel{\left[W_{\theta \sigma_{2}}^{\lambda}\right]}{\sim} D_{j k}$, then $B_{j k} \stackrel{W S_{\theta \sigma_{2}}^{\lambda}}{\sim} D_{j k}$.

(ii) If for each $y \in Y \sup _{j, k, s, t}\left|\mu_{y}\left(\frac{B_{\sigma^{j}(s) \sigma^{k}(t)}}{D_{\sigma^{j}(s) \sigma^{k}(t)}}\right)\right|<\infty$ and $B_{j k} \stackrel{W S_{\theta \sigma_{2}}^{\lambda}}{\sim} D_{j k}$, then $B_{j k} \stackrel{\left[W_{\theta \sigma_{2}}^{\lambda}\right]}{\sim} D_{j k}$

Proof. (i) Let $B_{j k} \stackrel{\left[W_{\theta \sigma_{2}}^{\lambda}\right]}{\sim} D_{j k}$. For every $\varepsilon>0$ and each $y \in Y$, we have

$$
\begin{aligned}
& \sum_{(j, k) \in I_{r u}}\left|\mu_{y}\left(\frac{B_{\sigma^{j}}(s) \sigma^{k}(t)}{D_{\sigma^{j}(s) \sigma^{k}(t)}}\right)-\lambda\right| \geq \sum_{(j, k) \in I_{r u}}\left|\mu_{y}\left(\frac{B_{\sigma^{j}(s) \sigma^{k}(t)}}{D_{\sigma^{j}(s) \sigma^{k}(t)}}\right)-\lambda\right| \\
& \left|\mu_{y}\left(\frac{B_{\sigma^{j}(s) \sigma^{k}(t)}}{D_{\sigma^{j}(s) \sigma^{k}(t)}}\right)-\lambda\right| \geq \varepsilon \\
& \geq \varepsilon\left|\left\{(j, k) \in I_{r u}:\left|\mu_{y}\left(\frac{B_{\sigma^{j}(s) \sigma^{k}(t)}}{D_{\sigma^{j}}(s) \sigma^{k}(t)}\right)-\lambda\right| \geq \varepsilon\right\}\right|
\end{aligned}
$$

for all $s, t$, which gives the result.

(ii) Let $B_{j k} \stackrel{W S_{\theta \sigma_{2}}^{\lambda}}{\sim} D_{j k}$. Also, suppose that $\sup _{j, k, s, t}\left|\mu_{y}\left(\frac{B_{\sigma^{j}(s) \sigma^{k}(t)}}{D_{\sigma^{j}(s) \sigma^{k}(t)}}\right)\right|<\infty$ for each $y \in Y$. Then, there exists an $M>0$ such that for each $y \in Y$

$$
\left|\mu_{y}\left(\frac{B_{\sigma^{j}(s) \sigma^{k}(t)}}{D_{\sigma^{j}(s) \sigma^{k}(t)}}\right)-\lambda\right| \leq M
$$

for all $j, k$ and $s, t$. Thus, for every $\varepsilon>0$ and each $y \in Y$ we have

$$
\begin{aligned}
& \frac{1}{h_{r u}} \sum_{(j, k) \in I_{r u}}\left|\mu_{y}\left(\frac{B_{\sigma^{j}(s) \sigma^{k}(t)}}{D_{\sigma^{j}(s) \sigma^{k}(t)}}\right)-\lambda\right| \\
& =\frac{1}{h_{r u}} \quad \sum_{(j, k) \in I_{r u}}\left|\mu_{y}\left(\frac{B_{\sigma^{j}}(s) \sigma^{k}(t)}{D_{\sigma^{j}}(s) \sigma^{k}(t)}\right)-\lambda\right| \\
& \left|\mu_{y}\left(\frac{B_{\sigma^{j}(s) \sigma^{k}(t)}}{D_{\sigma^{j}(s) \sigma^{k}(t)}}\right)-\lambda\right| \geq \varepsilon \\
& +\frac{1}{h_{r u}} \sum_{(j, k) \in I_{r u}} \quad\left|\mu_{y}\left(\frac{B_{\sigma^{j}}(s) \sigma^{k}(t)}{D_{\sigma^{j}}(s) \sigma^{k}(t)}\right)-\lambda\right| \\
& \left|\mu_{y}\left(\frac{B_{\sigma^{j}}(s) \sigma^{k}(t)}{D_{\sigma^{j}(s) \sigma^{k}(t)}}\right)-\lambda\right|<\varepsilon \\
& \leq \frac{M}{h_{r u}}\left|\left\{(j, k) \in I_{r u}:\left|\mu_{y}\left(\frac{B_{\sigma^{j}(s) \sigma^{k}(t)}}{D_{\sigma^{j}}(s) \sigma^{k}(t)}\right)-\lambda\right| \geq \varepsilon\right\}\right|+\varepsilon
\end{aligned}
$$

for all $s, t$, which gives the result. 
With a technique similar to that of Theorem 1 the following theorem can be proved.

Theorem 2.

(i) If $B_{j k} \stackrel{\left[W_{\sigma_{2}}^{\lambda}\right]}{\sim} D_{j k}$, then $B_{j k} \stackrel{W S_{\sigma_{2}}^{\lambda}}{\sim} D_{j k}$.

(ii) If for each $y \in Y \sup _{j, k, s, t}\left|\mu_{y}\left(\frac{B_{\sigma^{j}(s) \sigma^{k}(t)}}{D_{\sigma^{j}(s) \sigma^{k}(t)}}\right)\right|<\infty$ and $B_{j k} \stackrel{W S_{\sigma_{2}}^{\lambda}}{\sim} D_{j k}$, then $B_{j k} \stackrel{\left[W_{\sigma_{2}}^{\lambda}\right]}{\sim} D_{j k}$.

Theorem 3. If $\liminf _{r} q_{r}>1$ and $\liminf _{u} q_{u}>1$ for any $\theta_{2}=\left\{\left(j_{r}, k_{u}\right)\right\}$, then

$$
B_{j k} \stackrel{W S_{\sigma_{2}}^{\lambda}}{\sim} D_{j k} \quad \text { implies } B_{j k} \stackrel{W S_{\theta \sigma_{2}}^{\lambda}}{\sim} D_{j k}
$$

Proof. Let $B_{j k} \stackrel{W S_{\sigma_{2}}^{\lambda}}{\sim} D_{j k}$. Also, suppose that $\liminf q_{r}>1$ and $\liminf _{u} q_{u}>1$. Then, there exist $\eta, \rho>0$ such that $q_{r} \geq \eta+1, q_{u} \geq \rho+1$ for all $r, u>1$, which implies that

$$
\frac{h_{r u}}{j_{r} k_{u}} \geq \frac{\eta \rho}{(\eta+1)(\rho+1)} .
$$

Thus, for every $\varepsilon>0$ and each $y \in Y$ we have

$$
\begin{aligned}
\frac{1}{j_{r} k_{u}}\left|\left\{(j, k): j \leq j_{r}, k \leq k_{u},\left|\mu_{y}\left(\frac{B_{\sigma^{j}(s) \sigma^{k}(t)}}{D_{\sigma^{j}(s) \sigma^{k}(t)}}\right)-\lambda\right| \geq \varepsilon\right\}\right| \\
\quad \geq \frac{1}{j_{r} k_{u}}\left|\left\{(j, k) \in I_{r u}:\left|\mu_{y}\left(\frac{B_{\sigma^{j}(s) \sigma^{k}(t)}}{D_{\sigma^{j}(s) \sigma^{k}(t)}}\right)-\lambda\right| \geq \varepsilon\right\}\right| \\
\quad=\frac{h_{r u}}{j_{r} k_{u}} \frac{1}{h_{r u}} \mid\left\{(j, k) \in I_{r u}: \mid \mu_{y}\left(\frac{B_{\sigma^{j}(s) \sigma^{k}(t)}}{\left.\left.D_{\sigma^{j}(s) \sigma^{k}(t)}\right)-\lambda \mid \geq \varepsilon\right\} \mid}\right.\right. \\
\geq \frac{\eta \rho}{(\eta+1)(\rho+1)} \frac{1}{h_{r u}}\left|\left\{(j, k) \in I_{r u}:\left|\mu_{y}\left(\frac{B_{\sigma^{j}(s) \sigma^{k}(t)}}{D_{\sigma^{j}(s) \sigma^{k}(t)}}\right)-\lambda\right| \geq \varepsilon\right\}\right|
\end{aligned}
$$

for all $s, t$, which gives the result.

Theorem 4. If $\lim \sup _{r} q_{r}<\infty$ and $\lim \sup _{u} q_{u}<\infty$ for any $\theta_{2}=\left\{\left(j_{r}, k_{u}\right)\right\}$, then

$$
B_{j k} \stackrel{W S_{\theta \sigma_{2}}^{\lambda}}{\sim} D_{j k} \text { implies } B_{j k} \stackrel{W S_{\sigma_{2}}^{\lambda}}{\sim} D_{j k}
$$

Proof. Let $\lim \sup _{r} q_{r}<\infty$ and $\lim \sup _{u} q_{u}<\infty$. Then, there exist $\alpha, \beta>0$ such that $q_{r}<\alpha, q_{u}<\beta$ for all $r, u>1$. Also, suppose that $B_{j k} \stackrel{W S_{\theta \sigma_{2}}^{\lambda}}{\sim} D_{j k}$ and 
$\delta>0$. Then, there exist $n_{0}, m_{0} \in \mathbb{N}$ such that for every $\varepsilon>0$, each $y \in Y$ and all $j \geq n_{0}, k \geq m_{0}$

$$
\mathcal{S}_{j k}:=\frac{1}{h_{j k}}\left|\left\{(j, k) \in I_{j k}:\left|\mu_{y}\left(\frac{B_{\sigma^{j}(s) \sigma^{k}(t)}}{D_{\sigma^{j}}(s) \sigma^{k}(t)}\right)-\lambda\right| \geq \varepsilon\right\}\right|<\delta
$$

for all $s, t$. We can also find an $M>0$ such that $\mathcal{S}_{j k}<M$ for all $j, k=1,2, \ldots$.

Now, let $n$ and $m$ be any integers satisfying $j_{r-1}<n \leq j_{r}, k_{u-1}<m \leq k_{u}$ where $r>n_{0}, u>m_{0}$. Then, for every $y \in Y$ we have

$$
\begin{aligned}
& \frac{1}{n m}\left|\left\{(j, k): j \leq n, k \leq m,\left|\mu_{y}\left(\frac{B_{\sigma^{j}(s) \sigma^{k}(t)}}{D_{\sigma^{j}(s) \sigma^{k}(t)}}\right)-\lambda\right| \geq \varepsilon\right\}\right| \\
& \leq \frac{1}{j_{r-1} k_{u-1}}\left|\left\{(j, k): j \leq j_{r}, k \leq k_{u},\left|\mu_{y}\left(\frac{B_{\sigma^{j}(s) \sigma^{k}(t)}}{D_{\sigma^{j}(s) \sigma^{k}(t)}}\right)-\lambda\right| \geq \varepsilon\right\}\right| \\
&= \frac{1}{j_{r-1} k_{u-1}}\left|\left\{(j, k) \in I_{11}:\left|\mu_{y}\left(\frac{B_{\sigma^{j}(s) \sigma^{k}(t)}}{D_{\sigma^{j}(s) \sigma^{k}(t)}}\right)-\lambda\right| \geq \varepsilon\right\}\right| \\
&+\frac{1}{j_{r-1} k_{u-1}}\left|\left\{(j, k) \in I_{12}:\left|\mu_{y}\left(\frac{B_{\sigma^{j}(s) \sigma^{k}(t)}}{D_{\sigma^{j}(s) \sigma^{k}(t)}}\right)-\lambda\right| \geq \varepsilon\right\}\right| \\
&+\frac{1}{j_{r-1} k_{u-1}}\left|\left\{(j, k) \in I_{21}:\left|\mu_{y}\left(\frac{B_{\sigma^{j}(s) \sigma^{k}(t)}}{D_{\sigma^{j}(s) \sigma^{k}(t)}}\right)-\lambda\right| \geq \varepsilon\right\}\right| \\
&+\frac{1}{j_{r-1} k_{u-1}}\left|\left\{(j, k) \in I_{22}:\left|\mu_{y}\left(\frac{B_{\sigma^{j}(s) \sigma^{k}(t)}}{D_{\sigma^{j}(s) \sigma^{k}(t)}}\right)-\lambda\right| \geq \varepsilon\right\}\right| \\
& \vdots \\
&+\frac{1}{j_{r-1} k_{u-1}}\left|\left\{(j, k) \in I_{r u}:\left|\mu_{y}\left(\frac{B_{\sigma^{j}(s) \sigma^{k}(t)}}{D_{\sigma^{j}(s) \sigma^{k}(t)}}\right)-\lambda\right| \geq \varepsilon\right\}\right| \\
&= \frac{j_{1} k_{1}}{j_{r-1} k_{u-1}} \mathcal{S}_{11}+\frac{\left(j_{1}\left(k_{2}-k_{1}\right)\right.}{j_{r-1} k_{u-1}} \mathcal{S}_{12}+\frac{\left(j_{2}-j_{1}\right) k_{1}}{j_{r-1} k_{u-1}} \mathcal{S}_{21}+\frac{\left(j_{2}-j_{1}\right)\left(k_{2}-k_{1}\right)}{j_{r-1} k_{u-1}} \mathcal{S}_{22} \\
& \vdots \\
&+\frac{\left(j_{n_{0}}-j_{n_{0}-1}\right)\left(k_{m_{0}}-k_{m_{0}-1}\right)}{j_{r-1} k_{u-1}} \mathcal{S}_{n_{0} m_{0}} \mid
\end{aligned}
$$




$$
\begin{aligned}
& +\frac{\left(j_{r}-j_{r-1}\right)\left(k_{u}-k_{u-1}\right)}{j_{r-1} k_{u-1}} \mathcal{S}_{r u} \\
\leq & \left\{\sup _{1 \leq j, 1 \leq k} \mathcal{S}_{j k}\right\} \frac{j_{n_{0}} k_{m_{0}}}{j_{r-1} k_{u-1}}+\left\{\sup _{j \geq n_{0}, k \geq m_{0}} \mathcal{S}_{j k}\right\} \frac{\left(j_{r}-j_{n_{0}}\right)\left(k_{u}-k_{m_{0}}\right)}{j_{r-1} k_{u-1}} \\
\leq & M \frac{j_{n_{0}} k_{m_{0}}}{j_{r-1} k_{u-1}}+\delta \alpha \beta
\end{aligned}
$$

for all $s, t$, which gives the result.

\section{Theorem 5. If}

$1<\liminf _{r} q_{r} \leq \limsup _{r} q_{r}<\infty$ and $1<\liminf _{u} q_{u} \leq \limsup _{u} q_{u}<\infty$ for any $\theta_{2}=\left\{\left(j_{r}, k_{u}\right)\right\}$, then

$$
B_{j k} \stackrel{W S_{\theta \sigma_{2}}^{\lambda}}{\sim} D_{j k} \text { if and only if } B_{j k} \stackrel{W S_{\sigma_{2}}^{\lambda}}{\sim} D_{j k} .
$$

Proof. The proof is obvious from Theorem 3 and Theorem 4 .

With techniques similar to that of Theorem 3, Theorem 4 and Theorem 5 , the following theorems can be respectively proved.

Theorem 6. If $\liminf _{r} q_{r}>1$ and $\liminf _{u} q_{u}>1$ for any $\theta_{2}=\left\{\left(j_{r}, k_{u}\right)\right\}$, then

$$
B_{j k} \stackrel{\left[W_{\sigma_{2}}^{\lambda}\right]}{\sim} D_{j k} \quad \text { implies } B_{j k} \stackrel{\left[W_{\theta \sigma_{2}}^{\lambda}\right]}{\sim} D_{j k}
$$

Theorem 7. If $\lim \sup _{r} q_{r}<\infty$ and $\lim \sup _{u} q_{u}<\infty$ for any $\theta_{2}=\left\{\left(j_{r}, k_{u}\right)\right\}$, then

$$
B_{j k} \stackrel{\left[W_{\theta_{\sigma_{2}}}^{\lambda}\right]}{\sim} D_{j k} \text { implies } B_{j k} \stackrel{\left[W_{\sigma_{2}}^{\lambda}\right]}{\sim} D_{j k}
$$

Theorem 8. If

$1<\liminf _{r} q_{r} \leq \limsup _{r} q_{r}<\infty$ and $1<\liminf _{u} q_{u} \leq \limsup _{u} q_{u}<\infty$ for any $\theta_{2}=\left\{\left(j_{r}, k_{u}\right)\right\}$, then

$$
B_{j k} \stackrel{\left[W_{\theta \sigma_{2}}^{\lambda}\right]}{\sim} D_{j k} \text { if and only if } B_{j k} \stackrel{\left[W_{\sigma_{2}}^{\lambda}\right]}{\sim} D_{j k} .
$$

\section{Conclusion}

When $(\sigma(s), \sigma(t))=(s+1, t+1)$, from Definitions 11 16 we get the definitions of asymptotical almost equivalence, asymptotical strong almost equivalence, asymptotical almost statistical equivalence, asymptotical lacunary almost equivalence, asymptotical lacunary strong almost equivalence and asymptotical lacunary almost statistical equivalence in the Wijsman sense for double set sequences. So, the analogues of Theorem 1.8 can also be obtained between these definitions, which have not been appeared anywhere by this time. 
Author Contribution Statements The authors contributed equally to this work. All authors read and approved the final copy of this paper.

Declaration of Competing Interests The authors declare that they have no known competing financial interest or personal relationships that could have appeared to influence the work reported in this paper.

Acknowledgement The authors are thankful to the referees for making valuable suggestions leading to the better presentations of this paper.

\section{REFERENCES}

[1] Pringsheim, A., Zur theorie der zweifach unendlichen Zahlenfolgen, Math. Ann., 53(3) (1900), 289-321. https://doi.org/10.1007/BF01448977

[2] Móricz, F., Statistical convergence of multiple sequences, Arc. Math, 81(1) (2003), 82-89. https://doi.org/10.1007/s00013-003-0506-9

[3] Mursaleen, M., Edely, O. H. H., Statistical convergence of double sequences, J. Math. Anal. Appl., 288(1) (2003), 223-231. https://doi.org/10.1016/j.jmaa.2003.08.004

[4] Patterson, R. F., Savaş, E., Lacunary statistical convergence of double sequences, Math. Commun. 10(1) (2005), 55-61.

[5] Savaş, E., Patterson, R. F., Double $\sigma$-convergence lacunary statistical sequences, J. Comput. Anal. Appl., 11(4) (2009), 610-615.

[6] Patterson, R. F., Rates of convergence for double sequences, Southeast Asian Bull. Math., 26(3) (2003), 469-478. https://doi.org/10.1007/s10012-002-0469-y

[7] Wijsman, R. A., Convergence of sequences of convex sets, cones and functions, Bull. Amer. Math. Soc., 70(1) (1964), 186-188. https://doi.org/10.1090/S0002-9904-1964-11072-7

[8] Baronti, M., Papini, P., Convergence of Sequences of Sets, In: Methods of Functional Analysis in Approximation Theory (pp. 133-155), Birkhäuser, Basel, 1986.

[9] Nuray, F., Rhoades, B. E., Statistical convergence of sequences of sets, Fasc. Math., 49(2) (2012), 87-99. https://doi.org/10.3968/j.pam.1925252820120402.2264

[10] Beer, G., Wijsman convergence: A survey, Set-Valued Anal., 2(1) (1994), 77-94. https://doi.org/10.1007/BF01027094

[11] Nuray, F., Ulusu, U., Dündar, E., Lacunary statistical convergence of double sequences of sets, Soft Comput., 20(7) (2016), 2883-2888. https://doi.org/10.1007/s00500-015-1691-8

[12] Nuray, F., Ulusu, U., Lacunary invariant statistical convergence of double sequences of sets, Creat. Math. Inform., 28(2) (2019), 143-150. https://doi.org/10.37193/CMI.2019.02.06

[13] Nuray, F., Dündar, E., Ulusu, U., Wijsman statistical convergence of double sequences of sets, Iran. J. Math. Sci. Inform., 16(1) (2021), 55-64. https://doi.org/10.29252/ijmsi.16.1.55

[14] Nuray, F., Patterson, R. F., Dündar, E., Asymptotically lacunary statistical equivalence of double sequences of sets, Demonstratio Math., 49(2) (2016), 183-196. https://doi.org/10.1515/dema-2016-0016

[15] Ulusu, U., Dündar, E., Asymptotically $\mathcal{I}_{2}$-lacunary statistical equivalence of double sequences of sets, J. Ineq. Spec. Funct., 7(2) (2016), 44-56.

[16] Ulusu, U., Gülle, E., Wijsman asymptotical $\mathcal{I}_{2}$-statistically equivalent double set sequences of order $\eta$, Commun. Fac. Sci. Univ. Ank. Ser. A1 Math. Stat., 69(1) (2020), 854-862. https://doi.org/10.31801/cfsuasmas.695309

[17] Gülle, E., Ulusu, U., Wijsman asymptotical $\mathcal{I}_{2}$-lacunary statistically equivalence of order $\eta$ for double set sequences, J. Appl. Math. Inform., (in press) (2021). 
[18] Pancaroğlu, N., Nuray, F., Savaş, E., On asymptotically lacunary invariant statistical equivalent set sequences, AIP Conf. Proc., 1558(1) (2013), 780-781. https://doi.org/10.1063/1.4825609

[19] Ulusu, U., Nuray, F., On asymptotically lacunary statistical equivalent set sequences, $J$. Math., 2013(Article ID 310438) (2013), 5 pages. https://doi.org/10.1155/2013/310438 